\title{
Estudio de caso: Propuesta de directrices para la formulación de un plan de uso sostenible del recurso hídrico en la vereda Caney Alto en el municipio de Restrepo, Meta
}

Case study: Proposed guidelines for the formulation of a plan for sustainable use of the hydrical resource in the verge Caney Alto in the municipality of Restrepo, Meta 


\title{
Estudio de caso: Propuesta de directrices para la formulación de un plan de uso sostenible del recurso hídrico en la vereda Caney Alto en el municipio de Restrepo, Meta ${ }^{1}$ Case study: Proposed guidelines for the formulation of a plan for sustainable use of the hydrical resource in the verge Caney Alto in the municipality of Restrepo, Meta
}

\author{
Sandra Juliana Gutiérrez Manrique ${ }^{2}$, Mauricio Enrique Messa Cabrales ${ }^{3}$, Juan Pablo \\ Rodríguez Miranda ${ }^{4}$ \\ 2,3 Universidad de los Llanos, ${ }^{4}$ Universidad Distrital Francisco José de Caldas
}

Artículo recibido en julio de 2016; artículo aceptado en abril de 2017

Citación del artículo: Gutiérrez, S., Messa, M. \& Rodríguez, J. (2017). Estudio de caso: propuesta de directrices para la formulación de un plan de uso sostenible del recurso hídrico en la vereda Caney Alto en el municipio de Restrepo, Meta. I+D Revista de Investigaciones, 10 (2), julio - diciembre de 2017, pp. 6 - 13

\begin{abstract}
Resumen
Actualmente, la vereda Caney Alto presenta conflictos ambientales asociados a la intervención de su vegetación natural, a la afectación de la integralidad del ecosistema, a usos no compatibles del suelo, y, en algunos casos a la sobreutilización de estos. La zona también presenta afectaciones ambientales por el mal manejo del recurso hídrico debido a que esta vereda es un atractivo turístico local y regional, lo cual ha generado un aumento significativo en la demanda y presiones excesivas sobre calidad del agua. El presente artículo expone los lineamientos ambientales para contribuir al desarrollo sostenible del municipio de Restrepo, en especial de la vereda Caney Alto, mediante la propuesta de estrategias participativas y de control que involucren a los actores sociales e institucionales en la conservación y manejo adecuado de las fuentes hídricas en la microrregión de la vereda.
\end{abstract}

Palabras clave: uso sostenible, recurso hídrico, lineamientos.

\footnotetext{
Abstract

At present, the village of Caney Alto presents environmental conflicts associated with the intervention of its natural vegetation, affecting the ecosystem's integrality, unsustainable uses of the soil, and, in some cases, the overuse of these. The area also has environmental effects due to the mismanagement of the water resource because this path is a local and

1. Artículo de investigación, con enfoque cuantitativo, resultado de un proyecto de investigación terminado, Perteneciente al área de Ingeniería Ambiental, Potabilización del agua, Desarrollado en el grupo de investigación Aquaformat, Financiado por la Universidad Distrital Francisco José de Caldas, Bogotá D. C., Colombia.

2. Zootecnista. Especialista en Gestión Ambiental Sostenible, Universidad de los Llanos. Investigadora en formación, H-index=0. Correo electrónico: sandrajulianag@yahoo.com.

3. Profesional Comercio Internacional. Especialista en Gestión Ambiental Sostenible, Universidad de los Llanos. Investigadora en formación, H-index=0. Correo electrónico: mauromessac@gmail.com.

4. Rodríguez Miranda Juan Pablo Profesor Asociado. Facultad del Medio Ambiente y Recursos Naturales. Universidad Distrital Francisco José de Caldas. Grupo de investigación Aquaformat. Dirección Postal: Carrera. Este No 15 - 82. Avenida Circunvalar Venado de Oro. Bogotá D. C. Colombia. Orcid: 35797547900. Correo electrónico: jprodriguezm@udistrital.edu.co.
} 
SANDRA JULIANA GUTIÉRREZ MANRIQUE, MAURICIO ENRIQUE MESSA CABRALES, JUAN PABLO RODRÍGUEZ MIRANDA Estudio de caso: propuesta de directrices para la formulación de un plan de uso sostenible del recurso hídrico en la vereda Caney Alto en el municipio de Restrepo, Meta

regional touristattraction, which has generateda significantincrease in demandand excessivepressureson water quality. This article presents the environmental guidelines to contribute to the sustainable development of the municipality of Restrepo, especially the Caney Alto, through the proposal of participatory strategies and control that involve social and institutional actors in the conservation and proper management of water sources in the microregion of the path.

Keywords: sustainable use, water resources, guidelines.

Se entiende que el agua es un recurso renovable, su disponibilidad es muy variable según la ubicación en el territorio y también en algunos espacios es limitada, dado que en muchas regiones en diversas épocas del año sufren escasez severa (Blesa, 2012). Además de esto, existen otros factores tales como la contaminación, la erosión, la escorrentía, la salinización y actividades antrópicas (como el turismo) asociadas en su gran mayoría con las presiones de las poblaciones y el uso ineficiente del agua, que contribuyen a disminuir los recursos hídricos, tanto en cantidad como en calidad (Rodríguez, 2010; Cuello, 2016).

De acuerdo con el Geological Survey US (1997), para Suramérica existe un volumen de agua de $11760 \mathrm{~km}^{3}$ de agua por año, equivalentes al $25 \%$ de las reservas mundiales, es decir unos $36073 \mathrm{~m}^{3}$ por habitante año (para el periodo 1995 al 2025). Aunque a simple vista existe una cantidad adecuada de agua dulce para la región, también se presenta escasez en varias áreas de la región, ocasionando el llamado estrés hídrico generado por el consumo humano anual de 1000 a $2000 \mathrm{~m}^{3}$ (Rodríguez, 2010; León, 2016).

Según el estudio nacional del agua (Instituto de Hidrología, Meteorología y Estudios Ambientales [ldeam], 2010), Colombia posee un rendimiento hídrico de $63 \mathrm{l} / \mathrm{s} \mathrm{km}{ }^{2}$ (por regiones sería: Pacífico, $124 \mathrm{l} / \mathrm{s} \mathrm{km}^{2}$; Amazonía, $81 \mathrm{l} / \mathrm{s} \mathrm{km}^{2}$; Caribe, $55 \mathrm{l} / \mathrm{s} \mathrm{km}^{2}$; y Orinoquía, $55 \mathrm{l} / \mathrm{s} \mathrm{km}^{2}$ ), el cual supera ampliamente al promedio mundial $\left.(10 \mathrm{l} / \mathrm{s} \mathrm{km})^{2}\right)$ y al promedio latinoamericano (21

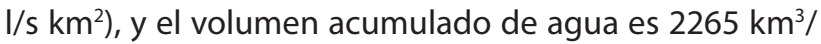
año distribuido por cuencas en $13 \%$ en la cuenca del Magdalena, 39\% en la Amazonía, $27 \%$ en la Orinoquía, $13 \%$ en la Pacífica, y $8 \%$ en la Caribe. Analizando la región de la Orinoquía, con base en los datos anteriores y en la población actual, se observa que el volumen de la oferta total accesible equivale a un total estimado de $500 \mathrm{~km}^{3}$ al año (GWP, 2009), y en esta misma región se presenta un rendimiento hídrico promedio de $55 \mathrm{l} / \mathrm{s} / \mathrm{km}^{2}$, mencionado anteriormente; este hecho hace evidente la inmensa riqueza del recurso hídrico que la región posee (Jara, 2007).

Otros elementos relacionados con la oferta hídrica regional que son significativos ocurren durante la temporada de lluvias intensas (aproximadamente nueve meses), cuando los rendimientos se acercan a los valores máximos $\left(70 \mathrm{l} / \mathrm{s} / \mathrm{km}^{2}\right)$; pero en la época seca los rendimientos pueden aproximarse, en algunas zonas, a los valores mínimos $\left(2,0 \mathrm{l} / \mathrm{s} / \mathrm{km}^{2}\right)$, mientras que el promedio es del orden de $55 \mathrm{l} / \mathrm{s} / \mathrm{km}^{2}$ (Jara, 2007).

La gestión ambiental en los recursos hídricos es un proceso que está orientado a prevenir, mitigar, controlar o compensar los problemas de carácter ambiental con el propósito de lograr un desarrollo sostenible, entendido este como aquel que le permite al hombre el desenvolvimiento de sus potencialidades y su patrimonio biofísico y cultural, además de garantizar la permanencia en el tiempo y en el espacio de los recursos hídricos (Baoying, 2007).

Por ello, se requiere conocimiento de lo que se va a administrar, solicita de la determinación de una visión futura deseable, definir los objetivos de conservación, preservación y aprovechamiento, y plantear propuestas de planeación que permitan su administración; a partir de su conocimiento se puede identificar y diseñar toda una serie de acciones, medidas, técnicas, mecanismos, proyecciones, herramientas y demás, que permitan, entre muchas otras cosas, identificar, prever y corregir los efectos de las intervenciones antrópicas, a la vez que solucionar, en la medida de lo posible, las afectaciones que ya existen (Gupta, 2005).

Según el Plan de Gestión Ambiental Regional (Gobernación del Meta, 2010), en el departamento del Meta, Colombia, se ha observado que existen diversos conflictos por el uso del suelo ( $35 \%$ presenta un uso pertinente, $48,8 \%$ presenta uso inadecuado, 14,3\% está subutilizado y $1,9 \%$ está sobre sobreutilizado con ganadería intensiva y cultivos tradicionales), en el cual también está asociado a un manejo inadecuado de los recursos hídricos.

En el municipio de Restrepo se capta y trata el agua del río Caney, que se distribuye al municipio de Cumaral; sin embargo, ésta también es utilizada para la porcicultura, la ganadería y la agricultura, actividades que generan contaminación difusa en este mismo cuerpo lótico. Otra presión antrópica que se ejerce sobre este recurso hídrico es la práctica del turismo rural en la vereda Caney Alto, donde funcionan aproximadamente nueve 
balnearios que abarcan un área de $2365 \mathrm{~m}^{2}$, y generan represamiento del agua debido a las piscinas construidas, hecho que afecta considerablemente el entorno tanto ambiental como social.

Actualmente, las autoridades del municipio de Restrepo no advierten la problemática real que sufre Caney Alto, dado que se puede presentar un impacto negativo tanto ambiental como económico debido a la densidad de población turística que puede presentarse por las aglomeraciones en la vereda, al igual que pueden presentarse conflictos de uso entre los usuarios tales como la infraestructura y los asentamientos, el turismo, la energía, la gestión del agua y la conservación de la naturaleza, los cuales pueden ser especialmente intensos, como se presenta en el área de estudio.

Desde luego que la creciente demanda de turismo rural es una gran oportunidad económica para los destinos turísticos bien desarrollados regionalmente, que por lo general está basado en el turismo local e inclusive regional para ampliar su oferta turística, así como para promover otros valores interesantes como el patrimonio cultural, local, etc., lo cual generaría una presión mayor hacia el recurso hídrico.

El turismo rural depende en gran medida del recurso hídrico dado que es un recurso necesario para prestar servicios básicos relacionados con necesidades (Marzelli, 2011) tales como condiciones sanitarias, higiene, salud pública y alimentación, así como una condición previa de ser un activo esencial para una amplia gama de la oferta turística y actividades (Gossling, 2011). El turismo rural por lo general mejora la economía y aumenta los beneficios de los destinos turísticos. Sin embargo, por lo común es un gran generador equívoco para el ambiente y la cultura, con muchos pro y contra para el medio debido al deterioro de la calidad del agua, que es cada vez más grave contra el desarrollo turístico pujante (Baoying, 2007).

De acuerdo con lo anterior, entre las principales causas del deterioro de la cuencas de cuerpos de agua lóticos (ecosistemas lóticos) están no manejar las cuencas de forma integral (GWP, 2009), la remoción de la vegetación, y los cambios en el uso de la tierra, que, en conjunto, pueden reducir la capacidad de retención de agua e incrementar la erosión causando una disminución en la disponibilidad de agua en las épocas secas y una mayor sedimentación en la cuenca baja (Fortuny, 2008). Otras de estas causas son los cambios en los flujos de los sedimentos y las cargas de contaminantes en los ríos, resultante de actividades lejanas (Andrade, 2004) así como de actividades actuales según el uso del suelo.
Por ello, los lineamientos ambientales para el uso sostenible del recurso hídrico en la vereda Caney Alto (apoyados en la herramienta de priorización de problemas mediante la matriz de Vester), son muy importantes, debido a las presiones antrópicas que se ejercen en el sector, dada la tendencia turística y el desarrollo económico de la zona de estudio razón por la cual se contribuye al desarrollo sostenible del municipio, mediante la construcción de estrategias participativas y de control que involucren a los actores sociales e institucionales en la conservación y manejo adecuado de las fuentes hídricas de la vereda Caney Alto.

\section{Método}

\section{Tipo de estudio}

El tipo de estudio es cualitativo, de corte descriptivo prospectivo (Vergel, 1997), dado que describe algunas características de la afectación del uso del cuerpo de agua dentro de un fenómeno homogéneo (Bryman, 2003), como lo es la vereda El Caney y su población beneficiada, el cual permite ubicar el análisis de las perspectivas de la investigación en el ámbito del cuerpo de agua, y encontrar los aspectos problemáticos en torno de un uso sostenible.

\section{Tipo de metodología utilizada}

La metodología propuesta para establecer los lineamientos ambientales en el uso sostenible del recurso hídrico se divide en las siguientes fases:

Fase 1. Diagnóstico. En esta fase se realizó un reconocimiento de la vereda Caney Alto; se recolectó información primaria y secundaria, con acercamiento a la comunidad para determinar aspectos relacionados con el uso del recurso hídrico en la vereda: Actividad n. ${ }^{\circ}$ 1. Visita de reconocimiento a la vereda Caney Alto con los actores de la comunidad; actividad n. ${ }^{\circ}$ 2. Identificación de actores dentro de la misma comunidad; actividad n..$^{\circ}$ 3. Conformación del grupo de trabajo; actividad n.. 4. Recopilación de información secundaria (bibliografía y cartografía); actividad n. ${ }^{\circ}$. Revisión y definición de las herramientas para el diagnóstico participativo; actividad n. ${ }^{\circ}$ 6. Levantamiento de información (secundaria) cartográfica de base necesaria para documentar el proyecto; actividad n. ${ }^{\circ}$. Socialización del diagnóstico y su complementación con la comunidad (taller participativo comunitario - cartografía social); actividad n. ${ }^{\circ}$ 8. Elaboración del documento de diagnóstico. Indicadores: informe de visita, asistencia comunidad, taller socialización y documento diagnóstico. 
SANDRA JULIANA GUTIÉRREZ MANRIQUE, MAURICIO ENRIQUE MESSA CABRALES, JUAN PABLO RODRÍGUEZ MIRANDA Estudio de caso: propuesta de directrices para la formulación de un plan de uso sostenible del recurso hídrico en la vereda Caney Alto en el municipio de Restrepo, Meta

Fase 2. Evaluación. A partir de la información obtenida en el diagnóstico, fase que permite conocer la problemática del uso del recurso hídrico, se identificaron y determinaron las causas y consecuencias de la situación problema, empleando herramientas de priorización de problemas como la matriz de Vester (Vester, 1983), y aplicada con éxito en diversos campos. Actividad n. ${ }^{\circ} 9$. Elaboración de la matriz; actividad n. ${ }^{\circ} 10$. Clasificación de los problemas. Indicadores: documento con el número de problemas y grado de importancia detectados.

Fase 3. Formulación. Gracias a las actividades de participación ciudadana y a la información recopilada, con apoyo de expertos, se plantearon los lineamientos para el uso del recurso hídrico en la vereda Caney Alto.

Fase 4. Socialización. Se puso en conocimiento de la comunidad en general la problemática actual de la oferta hídrica en la vereda y sus posibles alternativas de solución. Actividad n. ${ }^{\circ} 13$. Socialización a la comunidad de las propuestas de uso sostenible del recurso hídrico en la vereda; actividad $n .^{\circ} 14$. Elaboración de documento final. Indicadores: asistencia socialización, documento final impreso y medio digital.

Fase 5. Control y seguimiento. Para dar continuidad al objetivo del proyecto, se vinculó a los actores reguladores y comunitarios por medio de compromisos específicos para el control del uso y manejo sostenible de las fuentes hídricas de la vereda. Actividad n. 15. Firma del acta de compromiso por parte de los actores reguladores y comunitarios para dar continuidad al objetivo del proyecto. Indicadores: acta de compromiso firmada.

\section{Resultados}

Los resultados obtenidos durante el desarrollo del presente estudio son los siguientes:

- Se realizó un diagnóstico del uso del recurso hídrico con participación de la comunidad de la vereda Caney Alto, resultado de la sensibilización y socialización con la comunidad.

- Se identificaron y priorizaron los principales problemas que se presentan con el uso recurso hídrico en la vereda.

- Se formularon alternativas tendientes a solucionar la problemática encontrada.

- Con las informaciones obtenidas, se promovió la vinculación de los actores reguladores y comunitarios para crear mecanismos de control y manejo sostenible de las fuentes hídricas de la vereda.
Dichos resultados se obtuvieron aplicando la metodología de marco lógico (Ortengre, 2005), dada las bondades que este método ofrece (posibilita el diálogo entre las partes involucradas, identifica problemas soluciones, contribuye a clarificar y concretar objetivos del proyecto en función de las actividades, reduce la complejidad del proyecto y mejora las condiciones de relevancia, factibilidad y sostenibilidad del proyecto), y de las cuales se presentan los siguientes resultados (Tabla 1).

Es significativo señalar que en áreas con vocación turística rural como la vereda El Caney se debe tener un plan específico para valorar las posibilidades de ocio y recreo al aire libre. Dicha valoración tendrá en cuenta los servicios ambientales que presta el territorio y su acondicionamiento para las actividades e intervenciones propuestas, cuya justificación parte del aprovechamiento económico de los valores ecológicos y paisajísticos de la zona, el cual debe ser compatible con el análisis cognoscitivo del entorno de protección ambiental. (Gossling, 2011)

Según lo anterior, la protección ambiental de los recursos hídricos históricamente se ha realizado mediante el énfasis en la calidad y cantidad del agua, y no en la dinámica socio-ambiental de la zona de estudio como eje central para conservar la diversidad de especies nativas, la integridad ecológica en el cuerpo de agua y el uso del agua a lo largo de la cuenca. Pero también, con las acciones coordinadas para proteger y restaurar los impactos generados por la actividad turística rural, los cuales van directamente relacionados con las modificaciones del proceso hidrológico e hidráulico del cuerpo de lótico, la alteración de sus características morfológicas, las afectaciones sobre el hábitat de especies y, desde luego, los cambios sociales en el entorno (Fortuny, 2008).

Por ello, es importante disponer de un inventario del recurso hídrico (superficial y subterráneo) como herramienta esencial para fijar prioridades de acción en la zona de estudio. También es necesario diseñar el modelo de intervención para la conservación del recurso hídrico en función de un plan de manejo de dicho recurso (Gupta, 2005). En dicho plan se deben definir las acciones de protección del cuerpo de agua en términos de: adquisición de terrenos adyacentes para el desarrollo de un turismo sostenible; definición y delimitación de las zonas de protección; establecimiento de zonas destinadas a reforestación protectora productora; favorecimiento de la regeneración natural del ecosistema; diseño e implementación de un plan de acción para la actividad turística, y fortalecimiento de los compromisos de la comunidad. 
Tabla 1

Resultado del marco lógico en la vereda Caney Alto

\begin{tabular}{|c|c|c|c|c|c|}
\hline Fase & Objetivo & Actividad & Indicador & Participantes & Producto \\
\hline 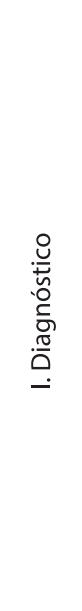 & $\begin{array}{l}\text { Elaborar un } \\
\text { diagnóstico del uso } \\
\text { del recurso hídrico } \\
\text { con participación de } \\
\text { la comunidad de la } \\
\text { vereda Caney Alto. }\end{array}$ & $\begin{array}{l}\text { Visita de reconocimiento a la } \\
\text { vereda Caney Alto. } \\
\text { Identificación de actores. } \\
\text { Conformación de grupo de } \\
\text { trabajo. } \\
\text { Recopilación de información } \\
\text { secundaria (bibliografía y } \\
\text { cartografía). } \\
\text { Revisión y definición de las } \\
\text { herramientas para el } \\
\text { diagnóstico participativo. } \\
\text { Elaboración de cartografía. } \\
\text { Socialización del diagnóstico y } \\
\text { su complementación con la } \\
\text { comunidad (taller de cartografía } \\
\text { social). } \\
\text { Elaboración del documento de } \\
\text { diagnóstico. }\end{array}$ & $\begin{array}{l}\text { Informes de visita. } \\
\text { Base de datos, habitantes de la } \\
\text { vereda: actores comunitarios e } \\
\text { institucionales. } \\
\text { Grupo de trabajo conformado. } \\
\text { Documentación: bibliografía, } \\
\text { cibergrafía, cartografía } \\
\text { existente. } \\
\text { Herramientas de diagnóstico } \\
\text { definidas. } \\
\text { Cartografía del área de } \\
\text { estudio. } \\
\text { Asistencia de la comunidad a la } \\
\text { socialización. } \\
\text { Documento diagnóstico. }\end{array}$ & $\begin{array}{l}\text { Grupo gestor. } \\
\text { Alcaldía municipal y } \\
\text { sus dependencias. } \\
\text { Comunidad de la } \\
\text { vereda Caney Alto. } \\
\text { Profesionales de apoyo } \\
\text { (biólogo). }\end{array}$ & 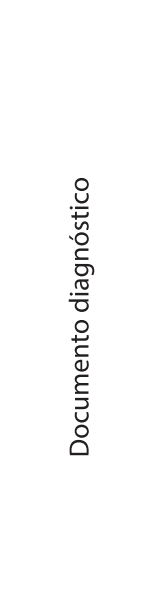 \\
\hline $\begin{array}{l}\stackrel{ }{0} \\
\frac{0}{U} \\
\frac{\pi}{2} \\
\frac{\pi}{2} \\
= \\
=\end{array}$ & $\begin{array}{l}\text { Identificar y } \\
\text { priorizar los } \\
\text { principales } \\
\text { problemas que se } \\
\text { presentan con el } \\
\text { uso recurso hídrico } \\
\text { en la vereda. }\end{array}$ & $\begin{array}{l}\text { Elaboración de la matriz. } \\
\text { Clasificación de los problemas. }\end{array}$ & $\begin{array}{l}\text { Matriz diseñada. } \\
\text { Problemas priorizados. }\end{array}$ & $\begin{array}{l}\text { Grupo gestor. } \\
\text { Profesionales de apoyo. } \\
\text { Comunidad vereda } \\
\text { Caney Alto. } \\
\text { Alcaldía municipal y sus } \\
\text { dependencias. }\end{array}$ & 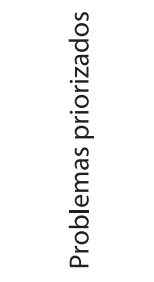 \\
\hline 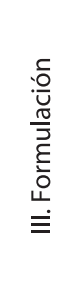 & $\begin{array}{l}\text { Formular } \\
\text { alternativas } \\
\text { tendientes a } \\
\text { solucionar la } \\
\text { problemática } \\
\text { encontrada, que } \\
\text { sirvan como } \\
\text { instrumentos de } \\
\text { planificación del } \\
\text { recurso hídrico y a } \\
\text { su uso sostenible. }\end{array}$ & $\begin{array}{l}\text { Recepción de aportes de } \\
\text { expertos. } \\
\text { Elaboración de la propuesta. }\end{array}$ & $\begin{array}{l}\text { Documento borrador, recepción } \\
\text { de aportes y alternativas. } \\
\text { Documento de la propuesta. }\end{array}$ & $\begin{array}{l}\text { Grupo gestor. } \\
\text { Profesionales Expertos. } \\
\text { Comunidad vereda } \\
\text { Caney Alto. } \\
\text { Alcaldía municipal y sus } \\
\text { dependencias. } \\
\text { UPNNS }\end{array}$ & 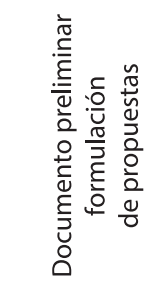 \\
\hline 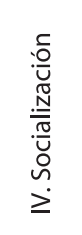 & $\begin{array}{l}\text { Socializar a la } \\
\text { comunidad en } \\
\text { general la } \\
\text { problemática actual } \\
\text { de la oferta hídrica } \\
\text { en la vereda y sus } \\
\text { posibles alternativas } \\
\text { de solución. }\end{array}$ & $\begin{array}{l}\text { Socialización a la comunidad de } \\
\text { las propuestas de uso sostenible } \\
\text { del recurso hídrico en la vereda. } \\
\text { Elaboración de documento final. }\end{array}$ & $\begin{array}{l}\text { Asistencia a la socialización. } \\
\text { Documento final. }\end{array}$ & $\begin{array}{l}\text { Grupo gestor. } \\
\text { Profesionales expertos. } \\
\text { Comunidad vereda } \\
\text { Caney Alto. } \\
\text { Alcaldía municipal y sus } \\
\text { dependencias. }\end{array}$ & 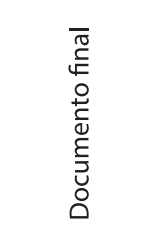 \\
\hline 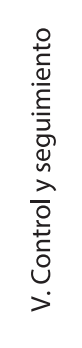 & $\begin{array}{l}\text { Vincular a los } \\
\text { actores reguladores } \\
\text { y comunitarios para } \\
\text { crear mecanismos } \\
\text { de control y manejo } \\
\text { sostenible de las } \\
\text { fuentes hídricas de } \\
\text { la vereda. }\end{array}$ & $\begin{array}{l}\text { Firma de compromiso por parte } \\
\text { de los actores reguladores y } \\
\text { comunitarios. }\end{array}$ & $\begin{array}{l}\text { Acta de compromiso de los } \\
\text { actores reguladores y } \\
\text { comunitarios de la vereda. }\end{array}$ & $\begin{array}{l}\text { Grupo gestor. } \\
\text { Profesionales expertos. } \\
\text { Comunidad vereda } \\
\text { Caney Alto. } \\
\text { Alcaldía municipal y sus } \\
\text { dependencias. }\end{array}$ & 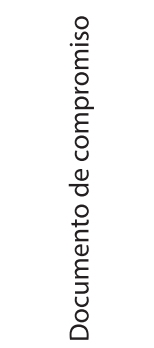 \\
\hline
\end{tabular}

\section{Fuente: Autores}


SANDRA JULIANA GUTIÉRREZ MANRIQUE, MAURICIO ENRIQUE MESSA CABRALES, JUAN PABLO RODRÍGUEZ MIRANDA Estudio de caso: propuesta de directrices para la formulación de un plan de uso sostenible del recurso hídrico en la vereda Caney Alto en el municipio de Restrepo, Meta

Todo lo anterior quiere decir que al proponer los lineamientos ambientales en la zona de estudio se pueden conseguir oportunidades que posibiliten una adecuada calidad de vida en el territorio estudiado. Desde luego, ello implica conservar y desarrollar los fundamentos del uso sostenible del recurso hídrico y también mantener en el largo plazo el potencial económico de la región. De acuerdo con lo anterior, se sugiere seguir profundizando en este tema mediante otro estudio de igual relevancia para la cuenca y que además incorpore la adaptación al cambio climático como variable de desarrollo.

\section{Conclusiones}

La posibilidad de construir estrategias participativas y de control que involucren a los actores sociales e institucionales en la conservación y manejo adecuado de las fuentes hídricas de la vereda Caney Alto se presenta como una estrategia oportuna para mantener el equilibrio de los balances y las sinergias entre las variables económicas, sociales y ambientales en la zona de estudio.

Siempre llevando consigo la participación de los actores locales con el propósito de potenciar sus compromisos y tomar decisiones relevantes en la dinámica del uso sostenible del recurso hídrico de la zona (valoración y acceso al agua), buscando crear conciencia y apropiación de la problemática del recurso hídrico regional, lo anterior debe ir acompañado de la acción de los actores reguladores y comunitarios para crear mecanismos de control para el manejo sostenible de las fuentes hídricas y también de la incorporación de dichos lineamientos ambientales en el Esquema de Ordenamiento Territorial de los municipios involucrados.

Finalmente, es importante establecer que se pueden definir los lineamientos ambientales para el uso del recurso hídrico en función de la demanda, oferta y calidad del agua en la zona de estudio; para ello, se debe determinar el estado actual (línea base) del recurso hídrico de la vereda mediante estudios adicionales que fortalezcan el conocimiento ambiental e hídrico de los cuerpos de aguas en la zona.

\section{Agradecimientos}

Los autores expresan sus más sinceros agradecimientos a la Coordinación del programa de maestría en Gestión Ambiental Sostenible de la Universidad de Los Llanos, así como al profesor de planta de la Universidad Distrital Francisco José de Caldas, Vidal Fernando Peñaranda Galvis por sus aportes y revisión de este documento.

\section{Referencias}

Andrade, P. (2004). Lineamientos para la aplicación del enfoque ecosistémico para la gestión integral de los recursos hídricos. Pnuma, 29 - 31.

Baoying, N. (2007). Tourism development and water pollution: casa study in lijang ancient town china population. Resources and environmetal, 123 - 127.

Blesa, M. (2012). Aguas naturales. Composición y características más importantes. En Agua y ambiente. Un enfoque desde la química. Buenos Aires: Peudeba.

Bryman, A. (2003). Social research methods. Second edition. New York: Oxford University Press.

Cuello, L. (2016). Determinación de la tasa relativa de crecimiento de la lemna minor sp en el tratamiento de efluentes de un sistema de tratamiento de aguas residuales municipales. I+D Revista de Investigaciones, 7(1), 91-97.

Fortuny, M. (2008). Technical approach for a sustainable tourism development. Case study in the balearica islands. Journal of cleaner production, $860-869$.

Gobernación del Meta. (2010). Plan de gestión ambiental regional. Villavicencio: Gobernación del Meta.

Gossling, S. (2011). Tourism and water use: supply, demand and security. An international review. Tourism management, 1 $-15$.

Gupta, J. (2005). Leegal reform for integrated water resources management a multi level dynamic. Incorporación de los principios de la gestión integrada de los recurso hídrico en los marcos legales de América Latina. Bogotá: Universidad Externado de Colombia.

GWP. (2009). Manual para la gestión integrada de recursos hídricos en cuencas. Londres: International network of basin organizations INBO.

Instituto de Hidrología, Meteorología y Estudios Ambientales. (2010). Estudio nacional del agua. Bogotá: Ideam.

Jara, A. (2007). Plan indicativo ambiental para el municipio de Restrepo, Meta. Villavicencio, Meta: Universidad de los Llanos.

León, K. (2016). Ecuaciones econométricas para los costos de inversión en plantas de tratamiento de agua potable en Colombia. I+D Revista de investigaciones, 7(1), 109-115.

Marzelli, S. (2011). Land resources in the alps and instruments supporting their sustainable management as a matter of regional environmental governance. Procedia social and behavioral sciencies, 141-155. 
Ortengre, K. (2005). Un resumen de la teoría que sustenta el metodo de marco lógico. Bogotá: ASDI.

Rodríguez, J. (2010). Contaminación del agua. Contaminación ambiental en Colombia. Bogotá: Fundación en causa por el desarrollo humano.

Vergel, G. (1997). Metodología. Un manual para la elaboración de diseños y proyectos de investigación. Barranquilla: Corporación Universitaria de la Costa. 